\title{
Accounting Information Systems and their Impact on Achieving Competitive Advantage
}

\author{
Anwar Hashim Abood \\ University of Al-Furat Al - Awsat, Musayab Technical Institute, Department of Accounting Techniques \\ anwar.2009@gmail.com
}

Keywords: Information Systems, National Islamic Bank, Iraqi Banks..

\begin{abstract}
This research aims to highlight the important and effective role played by accounting information systems in banking and its impact on the possibility of achieving competitive advantage. The collection, classification, analysis and submission of information to its users is one of the most important objectives that Iraqi banking departments seek to achieve under the acceleration of knowledge. By providing a robust database that is an effective tool for banks to achieve the principle of competitive advantage, these departments seek to develop their accounting information systems efficiently and effectively by employing their IT outputs in planning, The decision of the National Islamic Bank of Iraq as well as the design of a questionnaire consisting of 20 paragraphs specialized in accounting information systems and 40 questionnaires were distributed on the sample of the study. The role of accounting information systems in Iraqi banks and their impact on the achievement of competitive advantage. These indicators were analyzed and tested by means of the tests of F, P-value and impact statement through the R2 parameter using ANOVA. The research concluded that there is a significant effect on the systems. The accounting research to achieve the competitive advantage on the basis of which the alternative hypothesis was accepted. The research also reached a number of recommendations, the most important of which is that the departments of Iraqi banks are interested in investing and developing their accounting information systems and employing their information outputs in making the best administrative and investment decisions.
\end{abstract}

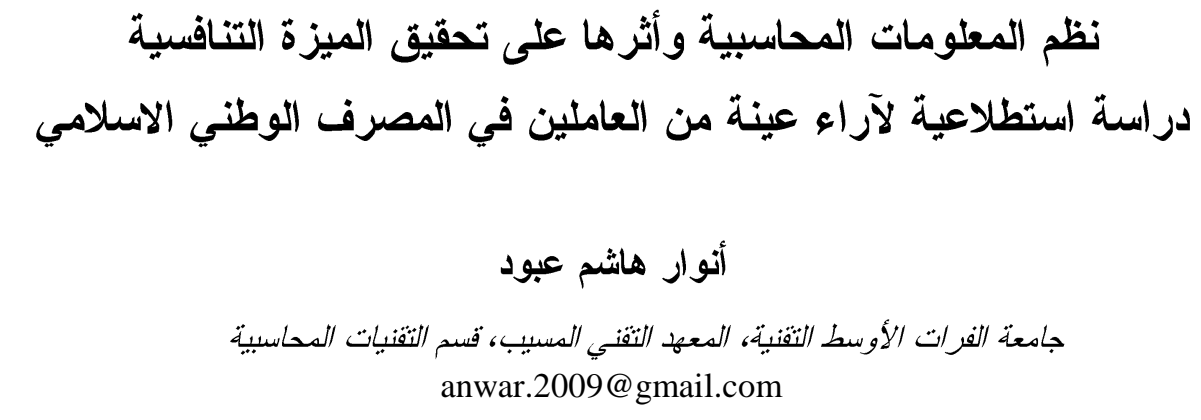

يهدف هذا البحث الى تسليط الضوء على الدور المهم والفعال الذي تؤديه نظم المعلومات المحاسبية في العمل المصرفي وتأثير ذلك علـى إمكانيــة

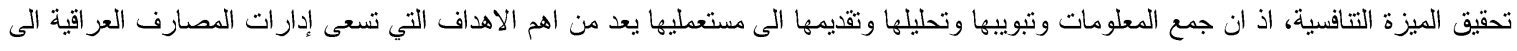

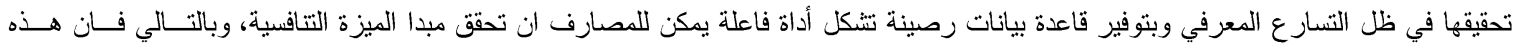


الإدارات تسعى تظوير نظم معلوماتها المحاسبية واستغلالها بكفاءة وفاعلية من خلال توظيف مخرجاتها المعلوماتية في عمليـات التخطــيط و التنظـيم

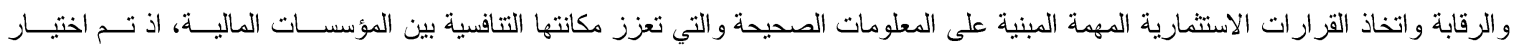

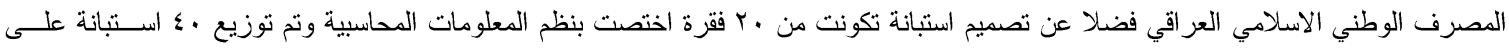

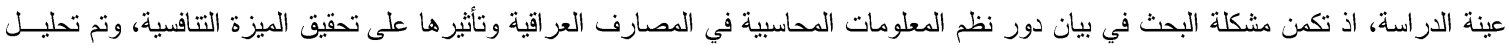

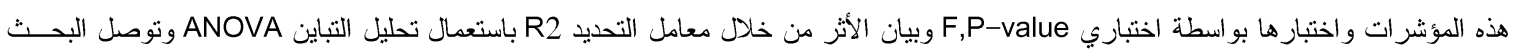

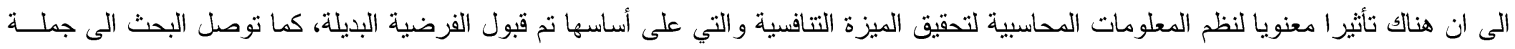

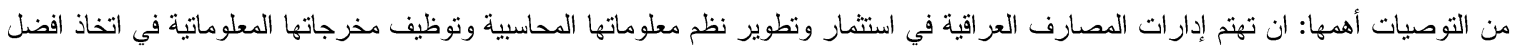

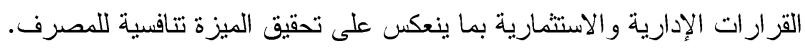

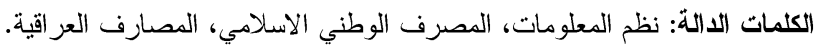

المقدمة

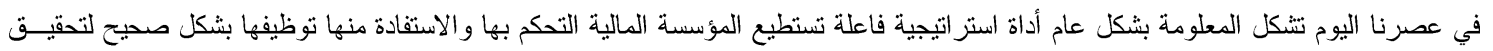

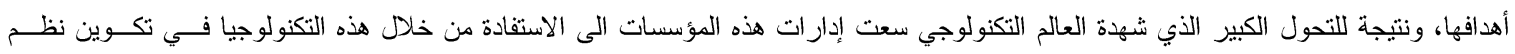

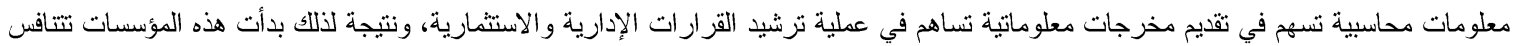
فيما بينها للسيطرة على الأسواق والتحكم بها غير ان هذا التحول اجبر هذه المؤسسات على تحمل أعباء مالية كبيرة لتكوين نظم معلوماتية محاسبية ذات

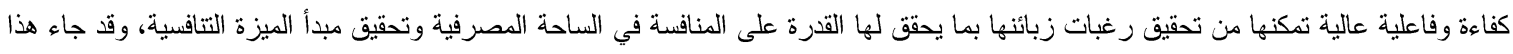

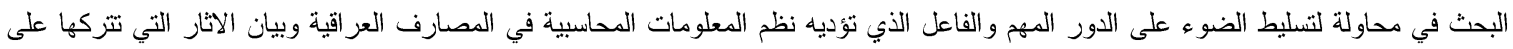

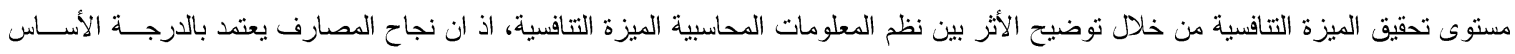

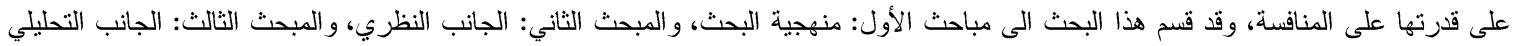

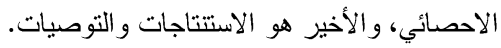

\section{1- منهجية البحث 1.1 مشكلة البحث}

ان توفير المعلومات المطلوبة من مختلف الجهات ذات العلاقة يحتاج الى جهودُ استثائية من قبل الوحدات الاقتصــادية، اذ ان تــوفير المعلومــة المطلوبة بكلفة اقل وبوقت قياسي يسهم في عملية اتخاذ القرار المحاسبي الصحيح وفي ظل المنافسة الثديدة بين الوحدات الاقتصادية ولاسيما المصرفية منها أصبح العالم يعي حقيقة ان امتلاك المعلومة من قبل هذه الوحدات هو ما يمكنها من تحقيق الميزة التتافسية مع الوحدات الأخرى فتقديم المعلومسـة فئة

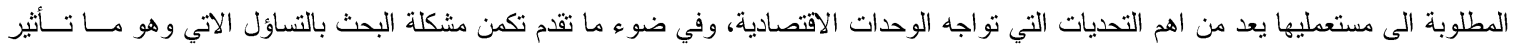

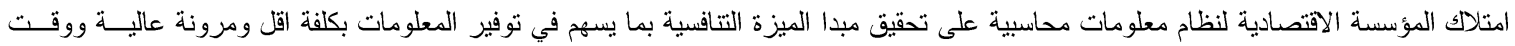

محدد.

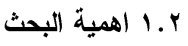

في ظل ما يشهده العالم اليوم من انفتاح معرفي ومعلوماتي أصبحت المعلومة المحاسبية ذا دور مهما وفاعل في النشاط المصرفي بشكل عام و النشاط المصرفي الإسلامي بشكل خاص، وكما ان انساع المنافسة بين الوحدات الاقتصادية على المستويين المحلي والدولي جعل منها نسعى الى توفير المعلومة

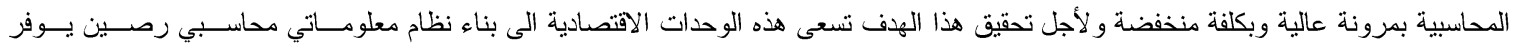

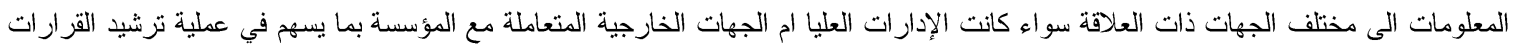

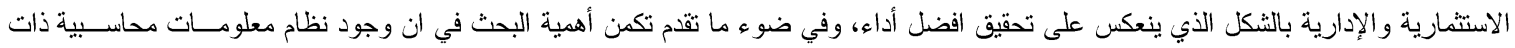

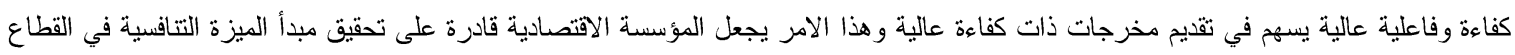
ا. تتاول المرتكزات المعرفية لكل من نظم المعلومات المحاسبية والميزة التنافسية.

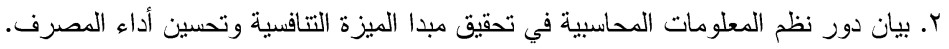
ع. التعرف على دور و اهمية نظم المعلومات المحاسبية في اداء المصرف دون. 


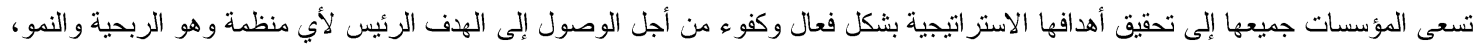

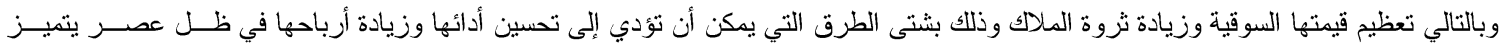

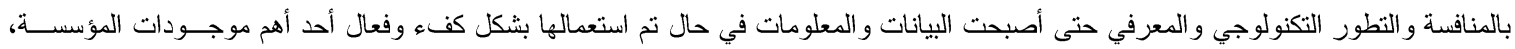

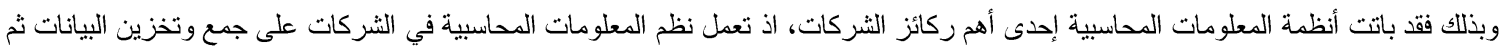

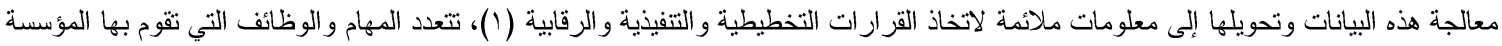

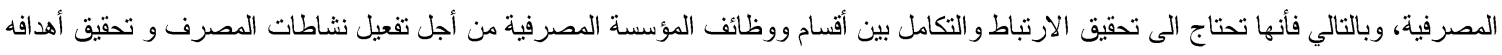

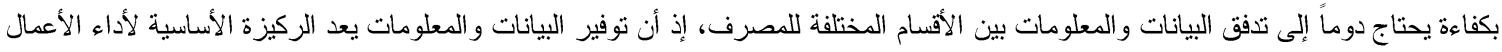

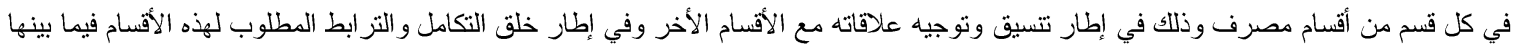

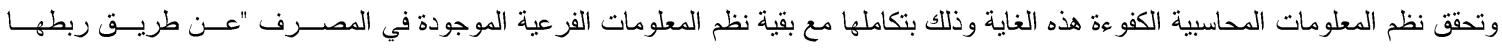

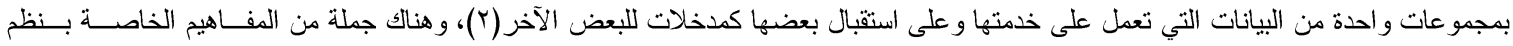

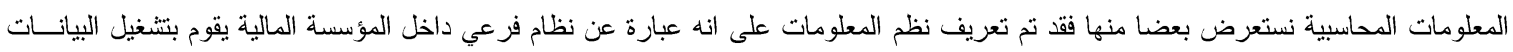

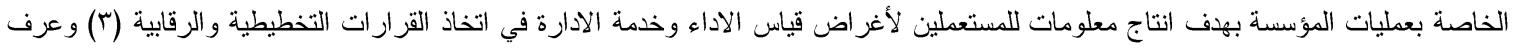

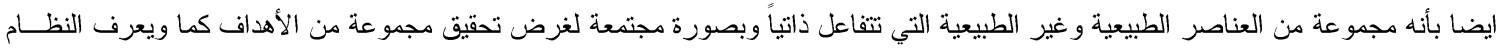

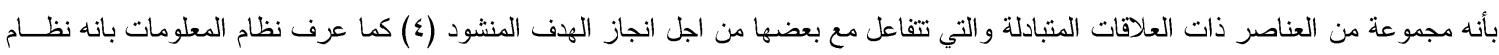

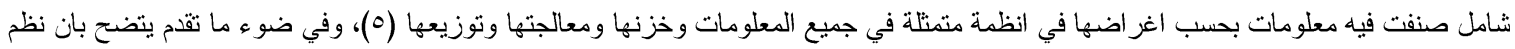

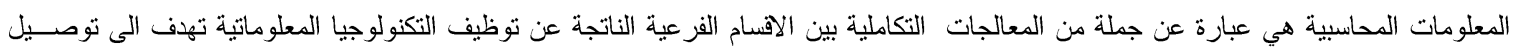
المعلومة المطلوبة الى مستخدميها عند الحاجة اليها أي ان هذه المعالجات تخص جملة كبيرة من الييانات لنحويلها الى معلومات ذات قيمة يلية يمكن الاعتماد عليها في عمليات اتخاذ القرار.

ب. r ب اهمية نظام المعلومات المحاسبية

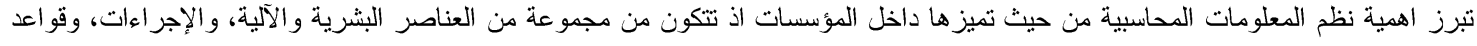

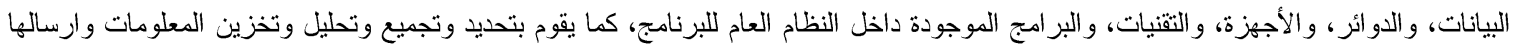

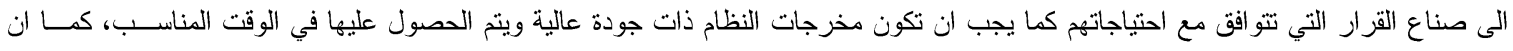

النظام يقوم بربط الهيكل التنظيمي اقسامه بجميعها (؟).

\section{r r.r خصائص نظم المعلومات المحاسبية}

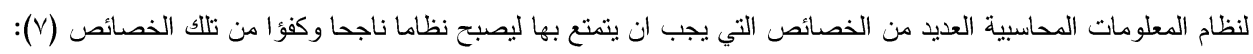

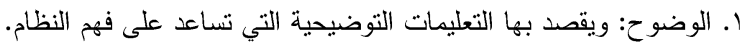

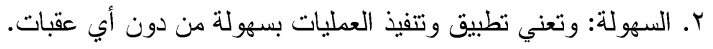

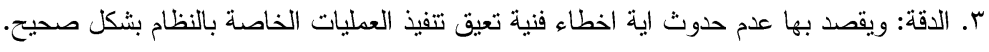

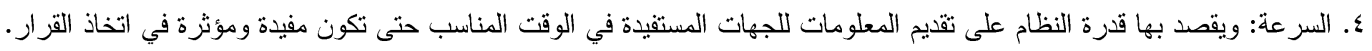

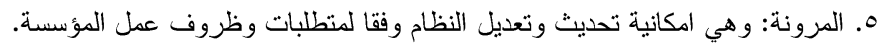

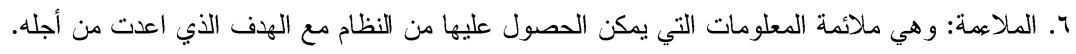

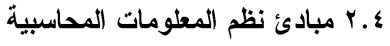
تعتمد كفاءة وفاعلية نظام المعلومات المحاسبية على مبادئ محددة وهي كالاتي:(^) 
ا. فاعلية التكلفة: يجب ان يكون النظام ألنكاليفي فاعلا، بمعني يجب ان تزيد المنافع المتوخاة من هذا النظام على التكاليف المتعلقة به.

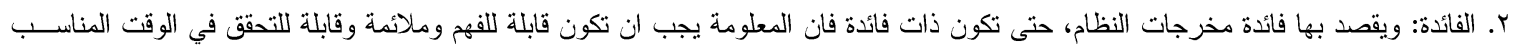
وموثوقة ويجب ان يأخذ مصممو نظام المعلومات بعين الاعنبار حاجة ومعرفة المستفيدين المختلفين من هذا النظام.

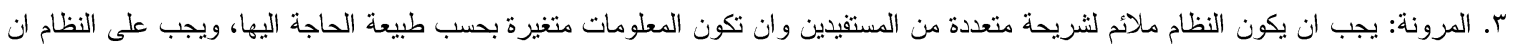

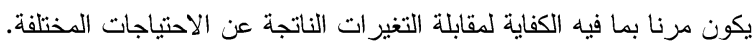

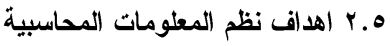

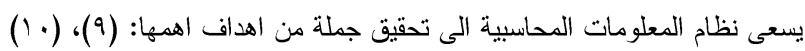

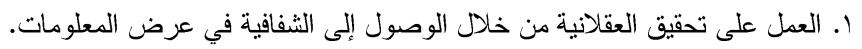
r. بـاعد في اتخاذ القرارات لكل الفاعلين في السوق. r. إعطاء معلومات صحيحة وكافية، موثوق بها وشفافة تتجع المستثمرين وتسمح لهم بمتابعة أمو الهم.

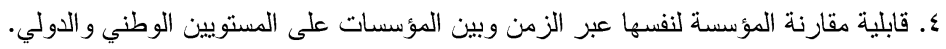

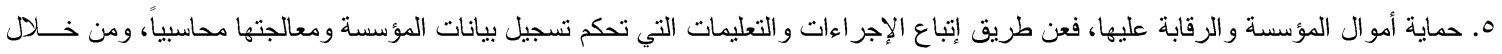

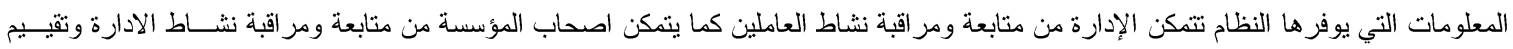
كفاءتها على سير اوجه النشاط كافة. T. المحافظة على المعلومات المدونة بشكل ثابت ومفهوم لدى مختلف العاملين بحقل المحاسبة.

تُمثل الميزة التتافية (Competitive Advantage) هدفاً رئيساً تسعى المؤسسات جميعها الى بلوغه لكي تثقى في السوق، وتستمر في

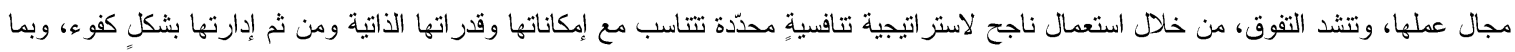

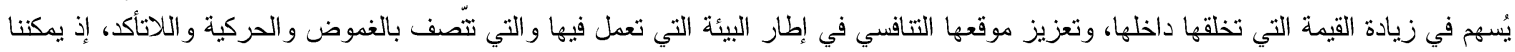

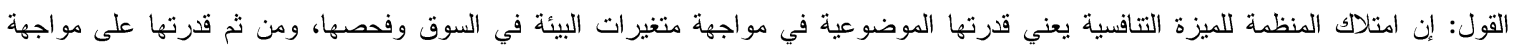

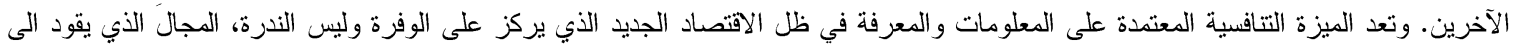

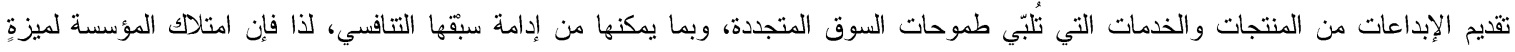
تتافسية يُعدّ دالةً لنجاح وتفوق المؤسسة في بيئتها، و إمكانية البقاء والاستمر ار فيها ستكون و اضحةً وجليّة [1 1]]، وفي ظل بيئات سريعة التغير تعيشها

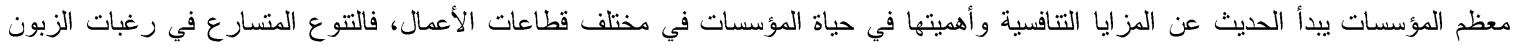

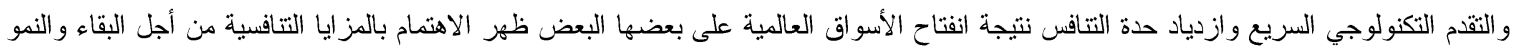

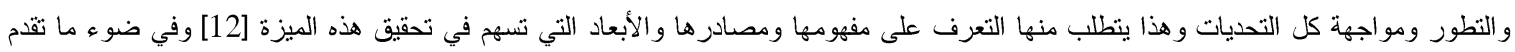

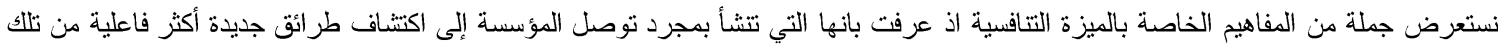

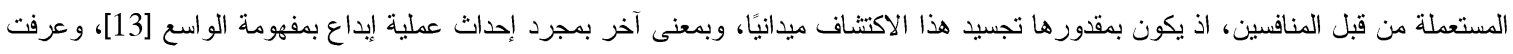
كذلك بانها المهارة او التقنية او المورد المتميز الذي يتيح للمؤسسة انتاج قيم ومنافع للزبائن تزيد عما يقدمه لهم المنافسون الاخرون ويؤكد ثميزها

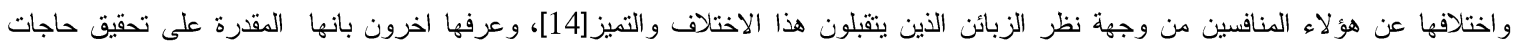

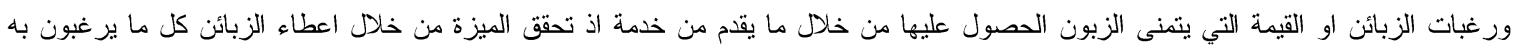
بصورة افضل من أبي جهة اخرى [15]، وتم تعريفها بانها الميزة في تميز المؤسسة على منافسيها بمركز فريد يتيح لها تقديم منتج متميز بأسلوب ناجح

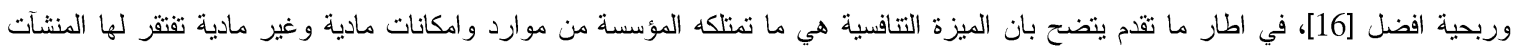
الاخزى بما يمكنها من تلبية الحاجات و الرغبات جميعها لدى زبائنها مما يجعلها قادرة على مو اجهة التقلبات الحاصلة في بيئة الاعمال.

تع الهمية الميزة التنافسية تعد الميزة النتافسية مهمة لأي منظمة في عالم اليوم وذلك للمو اكبة والاستمر ار في الانثطة والاعمال لذلك يمكن توضيح اهمية الميزة النتافسية بنقـاط عدة وهي كالاتي: [17] , [18] ا. انها الوسيلة التي يمكن من خلالها التفوق على المنافسين. r. انها تتبع من داخل المؤسسة وتحقق قيمة لها.

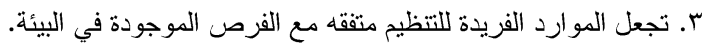
ع. قمكن للمنظمة من خلالها البقاء و النمو في بيئة شديدة المنافسة. 


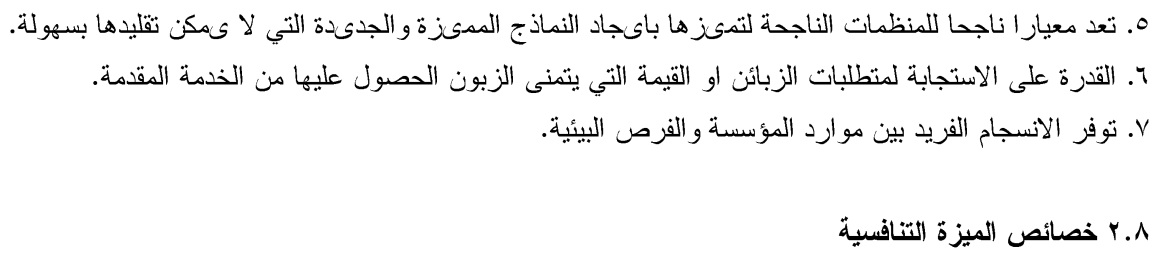
للميزة النتافسية مجموعة من الخصائص لا بد أن تتميز بها لتحقيق المنفعة للشركات مما ىمكنها من التفوق على منافسىها فئما تقدمه للزبائن لضمان

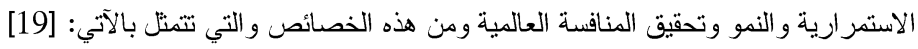

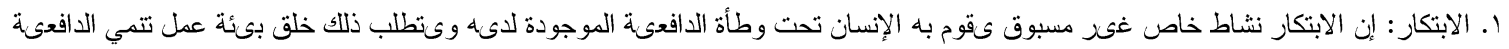

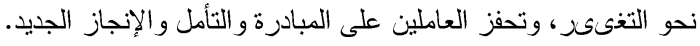

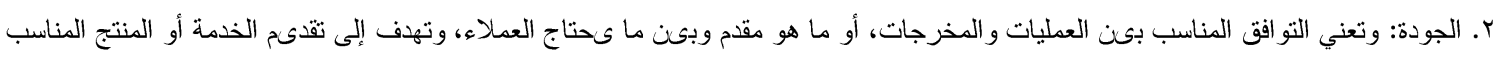
للعملاء وتخفض الخسائر ، والجودة لا تقوم على التوجهات الذاتية للمديرين. r. الكفاءة: وتعني القدرة على تحقيق الأهداف المحدة في زمن محدد مع مر اعاة جودة المخرجات، وهو من مقاىىس نجاح المؤسسات.

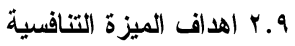

تسعى المؤسسة الاقتصادية ومن خلال اكتسابها للميزة التتافسية الى تحقيق الاتي: [20] ا. زيادة مستوى الارباح و الاخل للوحدة الاقتصادية ومن ثم زيادة قدرة الادخار مما يرفع من مستوى الاستثمار .

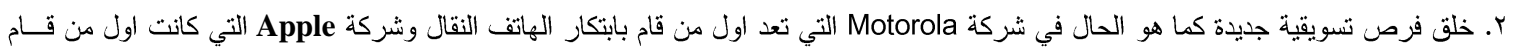
بابتكار الحاسب الثخصي. r. تكوين رؤية مستقبلية للأهداف التي تسعى المؤسسة الاقتصادية الى تحقيقها و الفرص التي ترغب في اقتتاصها. ع. التميز في الخدمة المقدمة من خلال تحسين خدمة التسليم او تحسين جودة الخدمة من خلال الجودة الاعلى للخدمة.

\section{r.l.}

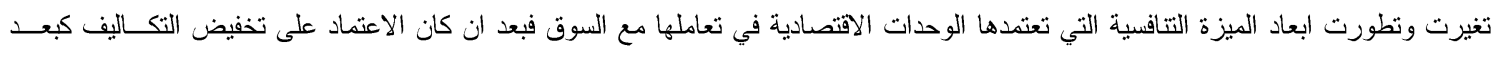

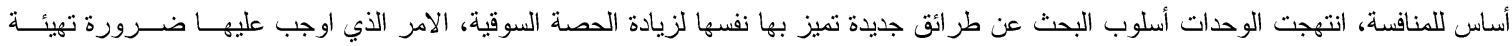

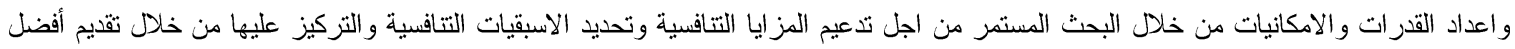

الخدمات التي تلبي حاجات ورغبات الزبائن و على ضوء هذه الاسبقيات تحدد الأهداف [21] ومن ابعاد الميزة التتافسية ما ياتي:

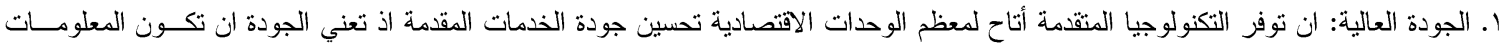

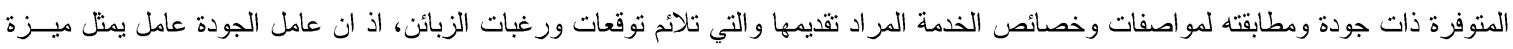

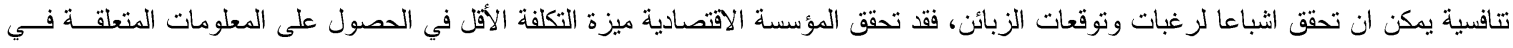

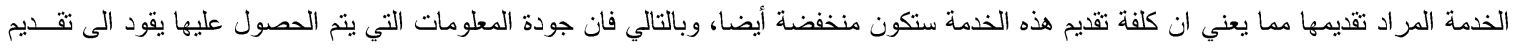

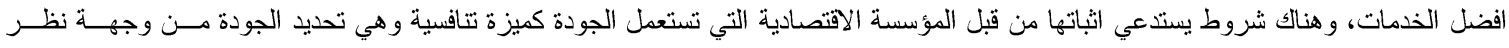
الزبائن وتجسيد سماتها الأساسية المرغوبة وتم ذلك من خلال الحصول على المعلومات ذات الجودة العالية [22]

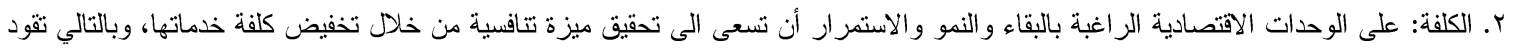

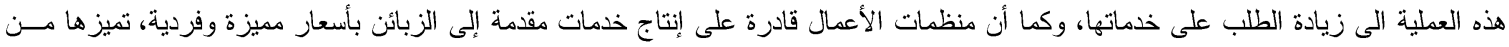

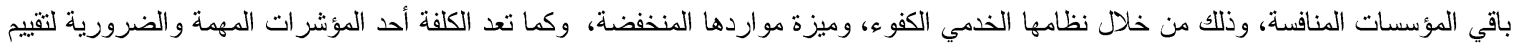

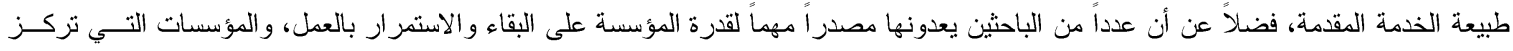

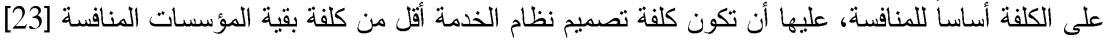

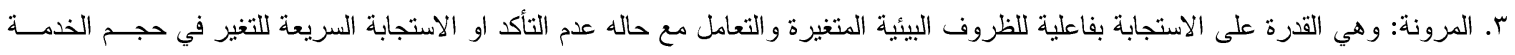

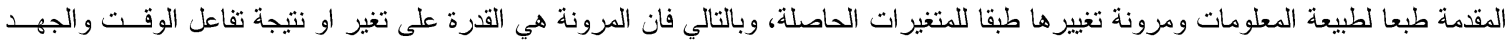

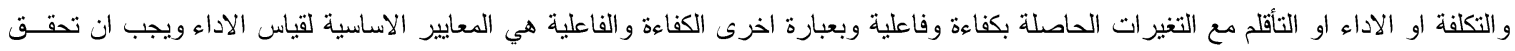

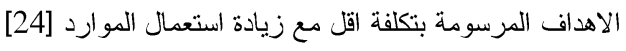


ع. وقت التسليم: إن اعتماد المنافسة على عامل الزمن أصبح من الضروريات لبناء الميزة التتافسية، وذلك بسبب التغيرات السريعة التي تجـري فـي التي

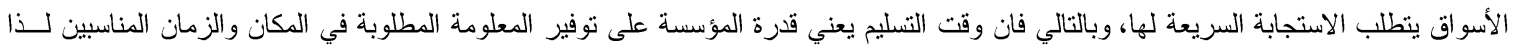

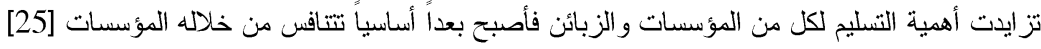

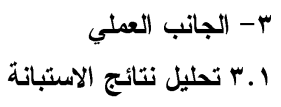

لأغر اض البحث فقد قمنا في تفريخ وتحليل البيانات من خلال برنامج (Easel) للقيام بعملية التحليل، وتحقيق الأهداف الموضوعة فـــي إطــار

البحث، وتم قياس صدق وثبات الاستبانة بمعامل الفا كرومباخ وذلك لغرض اختبار وقياس درجة المصداقية (الاعتمادية) في الاستجابات الواردة عـن لئن أسئلة الاستبانة، إذ يعتمد هذا الاختبار على مدى الثبات الداخلي ودرجة الاعتمادية لأسئلة الاسنبانة.

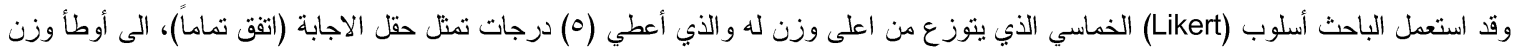

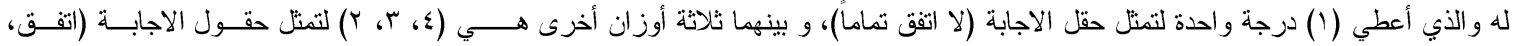
محايد، لا اتفق) على الترتيب، بعد ذلك تم عمل جداول التوزيع التكراري لكل محور من محاور نظم المعلومات المحاسبية، وتم اعداد الجداول الخاصة بعملية التحليل الاحصائي للحصول على الاوساط الحسابية الموزونة (C.V) (C.V) و الاوزان المئوية

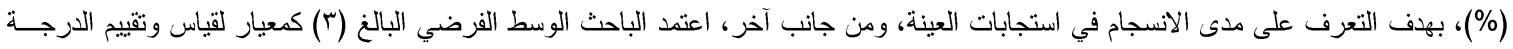

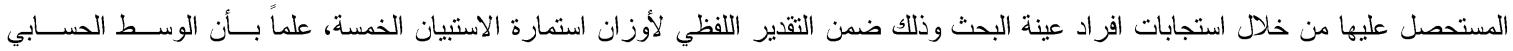

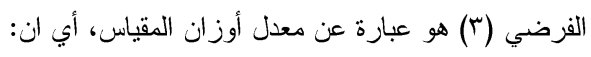

$$
3=\frac{1+2+3+4+5}{5}
$$

الجدول رقم (1) معامل ثبات محور نظم المعلومات المحاسبية.

\begin{tabular}{|c|c|c|}
\hline \multicolumn{3}{|c|}{ معامل الثبات (طريقة ألفا كرو نباخ) } \\
\hline معامل ألفا كرو نباخ للثبات & عدد الفقرات & 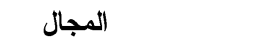 \\
\hline.$\wedge 7$ & r. & نظم المعلومات المحاسبية \\
\hline
\end{tabular}

الجدول رقم (ץ) فقرات الاستبانة/ محور نظم المعلومات المحاسبية ودورها في تفعيل أنشطة المصرف

\begin{tabular}{|c|c|c|c|c|c|}
\hline النسبي & الاختلاف معامل & الالحر اف المياري & الحسابي & 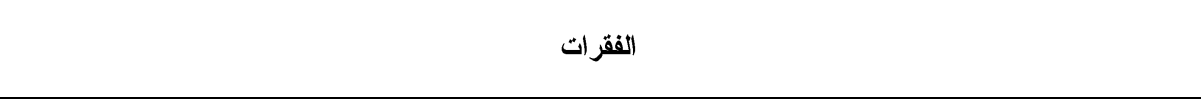 & $ت$ \\
\hline $84.00 \%$ & $13.81 \%$ &. .01 & 纟.r. & تحصل إدارة المصرف على معلومات صحيحة وفاعلة عند استعمال نظم المعلومات المحاسبية & 1 \\
\hline $79.50 \%$ & $27.42 \%$ & $1 . .9$ & r.91 & سهولة حصول الإدارة العليا على تقارير دورية مستمرة من خلال نظم المعلومات المحاسبية & r \\
\hline $81.50 \%$ & $21.84 \%$ &. .19 & $\varepsilon .+\Lambda$ & إمكانية تحقيق مبدأ الرقابة الااخلية على الأشطة المصرفية عذد الاعتماد على نظم معلومات محاسبية كفؤة & $r$ \\
\hline $86.50 \%$ & $17.57 \%$ &.$v 4$ & 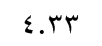 & تتعلم الأخطاء المحاسبية عند توظيف التكنولوجيا في اعداد المعلومات المحاسبية & $\varepsilon$ \\
\hline $86.50 \%$ & $16.88 \%$ &. $\mathrm{Vr}$ & L.r. & تتحقق ميزة ترشيد القرارات الإدارية و المالية المختلفة عند امتلاك المصرف نظم معلومات محاسبية كفؤة & $\bullet$ \\
\hline $86.50 \%$ & $15.95 \%$ &. .79 & L.r & تمكن مخرجات نظم المعلومات الجهات الخارجية الحصول على معلومات محاسبية صحيحة & 7 \\
\hline $84.00 \%$ & $17.14 \%$ &.$V Y$ & 纟.r. & تمنح نظم المعلومات المحاسبية الإدارة العليا ميزة تشخيص انحرافات الأداء بالاعتماد على مخرجاته من خلال الرقابة & $\checkmark$ \\
\hline $77.00 \%$ & $22.86 \%$ & $\cdot . \wedge \Lambda$ & r.^० & ان ترشيد ثرارات المستثمرين يعتمد بالدرجة الأساس على مخرجات نظم المعلومات المحاسبية & $\wedge$ \\
\hline $84.50 \%$ & $16.57 \%$ & $\because V$ & S.r & تمكن نظم المعلومات المحاسبية الإدارة العليا من تحقيق التكامل بين الأقسام والفروع المصرفية كافة & 9 \\
\hline $85.00 \%$ & $19.29 \%$ & $\cdot . \wedge r$ & 纟.ro & ان تكوين قواعد بيانات كبيرة يوفر من كلف الحصول على المطلومات المحاسبية المطلوبة & 1 . \\
\hline $83.50 \%$ & $18.93 \%$ & $\cdot . \wedge \vee$ & 4.17 & 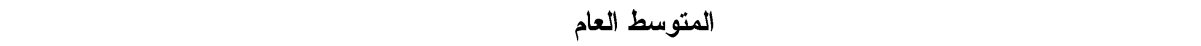 & \\
\hline
\end{tabular}




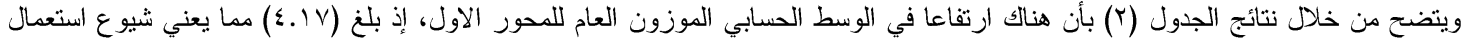

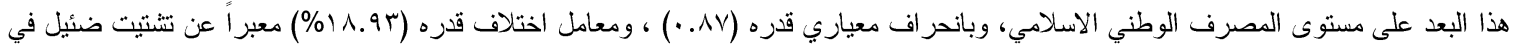

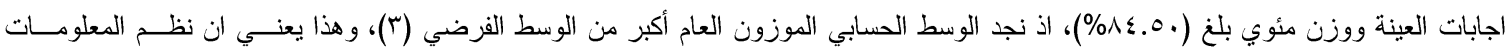

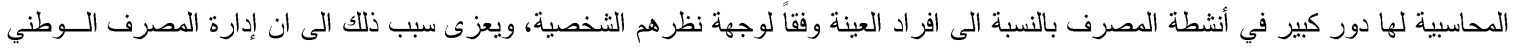

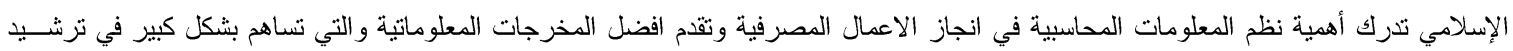

القرار ات الاستثمارية.

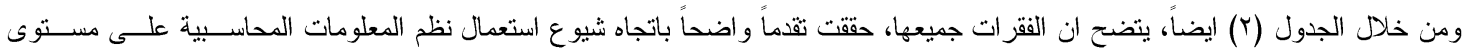

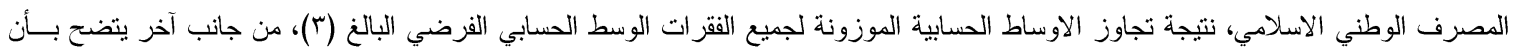
افر اد العينة منفقين بشكل كبير حول فقرات المحور الاول بنسب مئوية مرتفعة وتعد الفقرات (1) من أكثر الفقرات انسجاماً على مستوى اجابات العينة

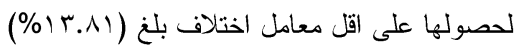

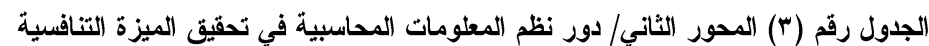

\begin{tabular}{|c|c|c|c|c|c|}
\hline المزن & الاختلاف معل & 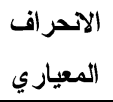 & الحسابي & 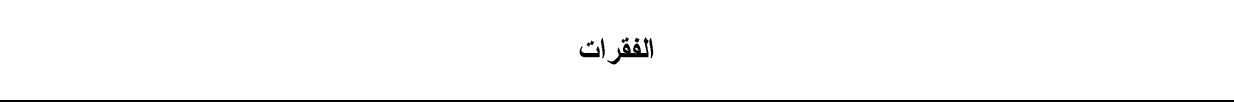 & $ت$ \\
\hline $80.00 \%$ & $21.00 \%$ &..$\wedge \varepsilon$ & $\varepsilon \ldots$ & تحقق الميزة التنافسية للمصرف عند امتلاكه معلومات ذات جودة عالية & 1 \\
\hline $79.00 \%$ & $20.00 \%$ &.$\vee \vee 9$ & r.90 & ان جودة مخرجات نظم المعلومات المحاسبية تسهم في اعداد تقارير مالية مبنية وفق المعايير المحاسبية الدولية & r \\
\hline $82.00 \%$ & $20.49 \%$ &..$\wedge \varepsilon$ & «.1. & ان قدرة المصرف على اعداد موازنات تخطيطية بالاعتماد على مخرجات نظم المعلومات المحاسبية تحقق ميزة تنافسية & $r$ \\
\hline $86.50 \%$ & $13.41 \%$ &. .01 & 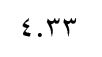 & وصول المعلومة المحاسبية المطلوبة في الوقت المناسب يحقق ميزة تنافسية & $\varepsilon$ \\
\hline $82.00 \%$ & $19.01 \%$ &.$\vee \wedge$ & «.1. & ان سرعة ودقة معالجة مدخلات نظم المعلومات المحاسبية يحقق للمصرف ميزة تنافسية في توصيل المعلومة في وقتها & $\bullet$ \\
\hline $79.00 \%$ & $18.99 \%$ &. . vo & r.90 & توفر نظم المعلومات المحاسبية ميزة تقليل الجها البشري في اعداد التقارير المطلوبة مما يسهم في تحقيق ميزة تنافسية & 1 \\
\hline $83.00 \%$ & $18.55 \%$ &.$V V$ & $\{.10$ & تحقى نظم المعلومات المحاسبية ميزة المرونة في تعديل التقارير المطلوبة حسب الحاجة اليها وفقا للمتغيرات البيئية & $v$ \\
\hline $74.00 \%$ & $21.62 \%$ &. .1 & r.v. & ان سهولة فهم واستعمال مخرجات نظم المعلومات المحاسبية من قبل الجهات المتعاملة يحقق للمصرف ميزة تنافسية & $\wedge$ \\
\hline $78.00 \%$ & $19.49 \%$ &.$V Y$ & ч. . . & ان توفرانخفاض كلف الحصول على المطومات المطلوبة من قبل الجهات الدائنة للمصرف يحقى ميزة تنافسية & 9 \\
\hline $76.00 \%$ & $19.21 \%$ &.$V r$ & r.А. & تحقق نظم المعلومات المحاسبية ميزة التغذية العكسية للذدمات المقدمة مما يسهم في تحقيق ميزة تنافسية & 1. \\
\hline $79.95 \%$ & $19.18 \%$ &. .84 & $\varepsilon \ldots$ & المتوسط العام & \\
\hline
\end{tabular}

ويتضح من خلال نتائج الجدول (r) بأن هناك ارتفاعا في الوسط الحسابي الموزون العام للححور الاول، إذ بلغ ( ...؛)، وبانحر اف معياري قدره

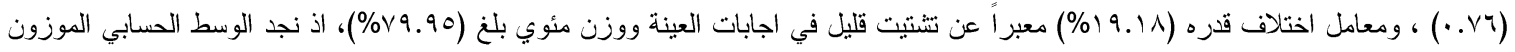

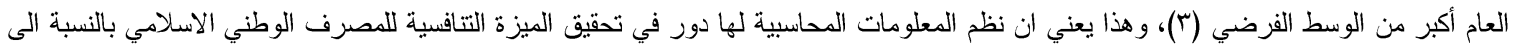
افراد العينة وفقاً لوجهة نظرهم الثخصية، ويعزى سبب ذلك الى ان إدارة المصرف الوطني الإسلامي تدرك ان تحقيق الميزة التتفسية يعد مقياسا لكفاءتها وفاعليتها وبالتالي فأنها تبحث عن مختلف الوسائل التي من شانها ان تحقق لها الغاية الأساسية من وجود المصرف وهي تقديم افضل الخدمات 
المصرفية اللى مختلف الزبائن المتعاملين بما يلبي حاجاتهم ورغباتهم، ومن ثم فأنها تولي أهمبة الى نظم المعلومات المحاسبية في انجاز الاعمال المصرفية.

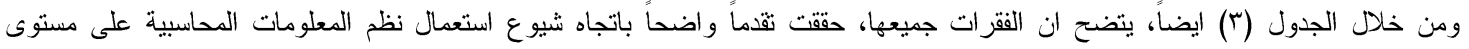

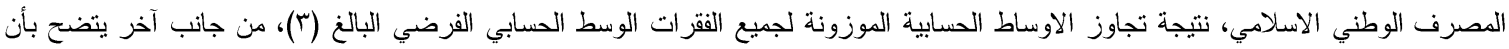
افراد العينة متفقين بشكل كبير حول فقرات المحور الثاني بنسب مئوية مرتفعة وتعد الفقرة (ع) من أكثز الفقزات انسجاماً على مستوى اجابات العينة

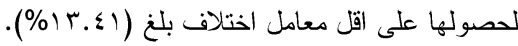

في ضوء ما تقدم ووفقا لتحليل إجابات افراد العينة يتضح بان لنظم المعلومات المحاسبية دورا مهما في عملية تحقيق الميزة التتافسية من خلال

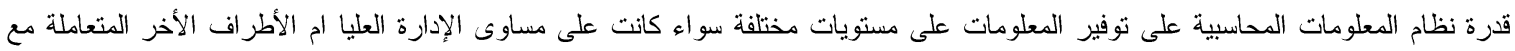

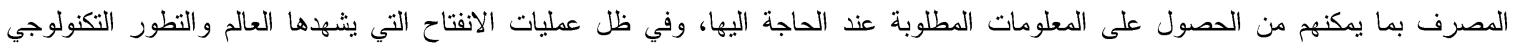
المتسارع أصبح للمعلومة المحاسبية أهمية كبيرة في الاعمال المصرفية فالقرارات المتخذة جميعها من قبل الإدارات العليا تستتد على المعلومات التي وفي يوفر ها هذا النظام بالتالي فان توفير المعلومة الصحيحة يسهم في اتخاذ القرارات الصحيحة التي تحقق الأهداف المطلوبة او المرسومة.

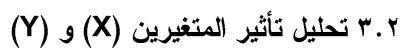

الجدول رقم (؛) تحليل تأثير بين المتغيرين (X) و (Y) ( الجنغر)

\begin{tabular}{|c|c|c|c|c|}
\hline \multicolumn{3}{|c|}{ X المتغير المستقل/ نظم المعلومات المحاسبية } & \multirow{2}{*}{ المتغير المعتمد } & \multirow{2}{*}{ اسم المصرف } \\
\hline p-value & F-test & $\mathbf{R}^{2}$ & & \\
\hline $0 . \ldots$ & $\varepsilon 0 . \vee \wedge$ & $0 . \wedge r$ & الميزة التنافسية & الوطني الإسدلامي \\
\hline
\end{tabular}

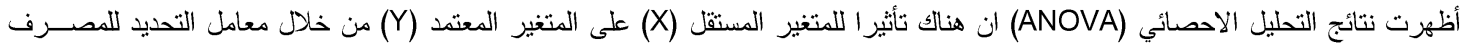

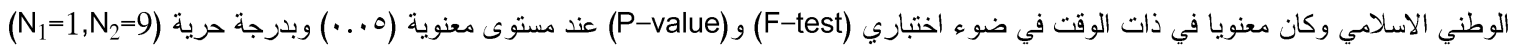

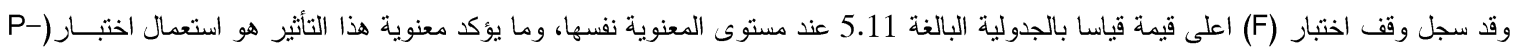

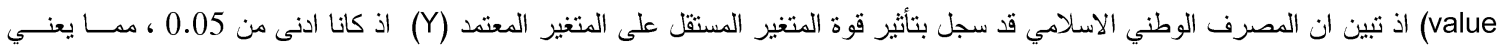
معنوية هذا التأثير.

؛ - الاستنتاجات و التوصيات

1 الاستنتاجات

ا. ان ضعف التشريعات و التعليمات التي تحكم ونتظم البيئية المصرفية العر اقية يجعل هناك صعوبة في العمل من جهة ويضعف قـدرة إدار ات هـذهـ المصارف على تحقيق النتافس فيما بينها بشكل صحيح. r. هناك توجه واستعداد لاى إدارة مصرف ايلاف الإسلامي على تبني فكرة تطوير نظم معلوماتها المحاسبية بالثكل الذي يجعلها ذات كفاءة وفاعليــة أكثر وبالتالي امتلاكه لقاعدة معلومات مفيدة مما يحقق الميزة التتافسية.

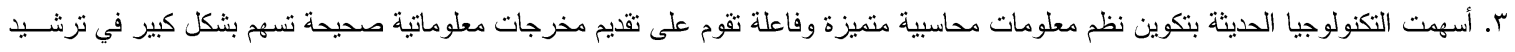

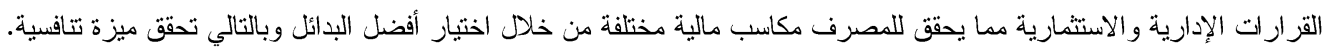

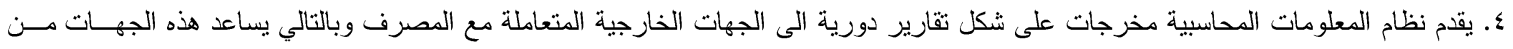

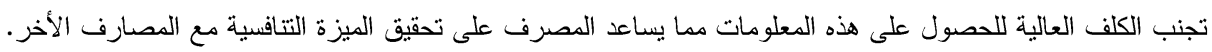

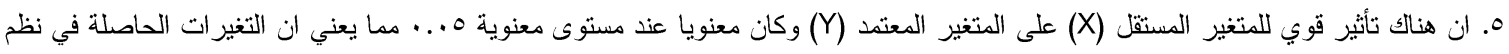

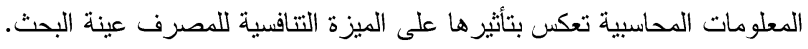


ا. ضرورة ان تتبني المصارف العر اقية فكرة عقد العديد من الورش الفنية التي من شانها ان تضع التعليمات والتشريعات التي نتظم المعل المصــرفي بشكل صحيح حتى تتمكن مصارفنا من المنافسة على المستوى المحلي و الدولي. r. ضرورة ان تقوم إدارات المصارف العر اقية على تطوير نظم معلوماتها المحاسبية من خلال ادخال أفضل الاجهزة البرمجيات الخاصة بهذا المجال

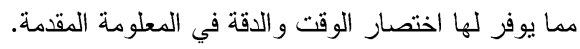
r. ضرورة ان تهتم المصارف العراقية بتظوير كوادرها البشرية العاملة على هذه الأنظمة من خلال المشاركة في الدورات المحلية والدولية مما يسهر في اكساب هؤلاء مهار ات فنية تخدم اهداف المصارف العان العاملين فيها.

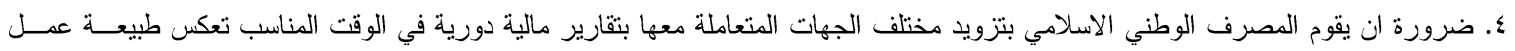
المصرف كون ذلك يساعد الجهات الخارجية على ثقييم و اقع المصرف من اجل اتخاذ القزارات الصحيحة مما يعزز و اقعه التتافسي فضلا عن فئل ان نظم CONFLICT OF INTERESTS

$$
\text { المعلومات نسهم في التتبؤ في الاداء المستقبلي. }
$$

There are no conflicts of interest.

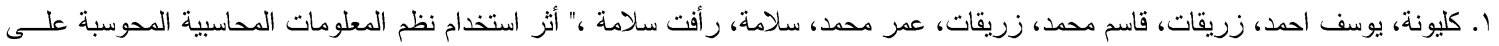

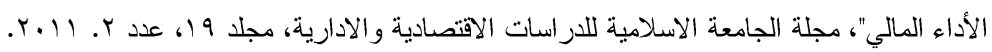

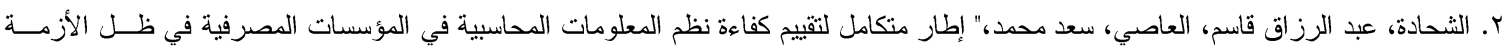

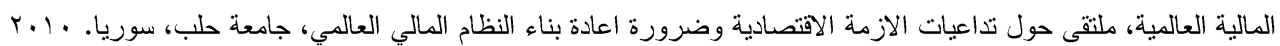

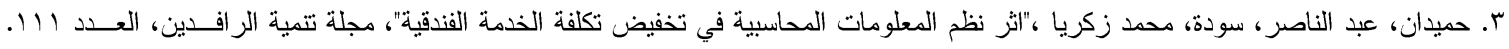
r. 11 ع. الر اوي، عبد اللطيف شهاب، أرسلان، إبر اهيم الافندي،" اثز الاستثمار في تقانة المعلومات وفاعلية نظام المعلومات المحاسبية في ظــل الاقتصــاد

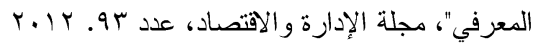

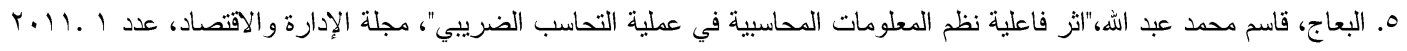
6. Al-Zeaud, Hussein" Accounting Information Systems and Their Role in the Measurement and Cost Thrifting in Public Shareholding Industrial”, International Journal of Business and Management. 2012.

V. مقداد، سعيد فتحي،" اثز مثاركة المحاسبين في تطوير نظم المعلومات المحاسبية على تحسين الأداء المالي"، رسالة ماجستير مقدمة الى مجلس كلية

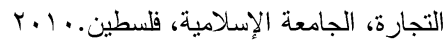

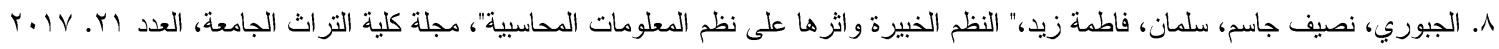

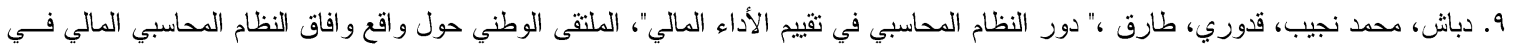

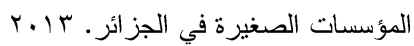
• 1 ـ بوحايك، عبد الباسط ،" دور نظام المعلومات المحاسبية في تحقيق فعالية الرقابة الداخلية للمؤسسة البترولية"، رسالة ماجستير مقدمة الى مجلس البس

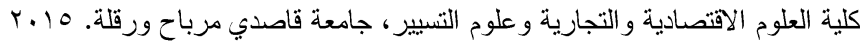

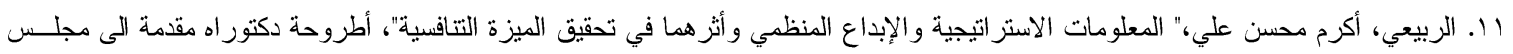

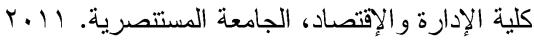

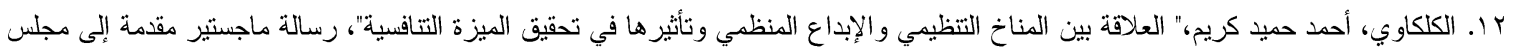

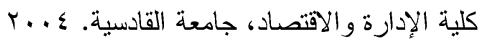
r ا. إِماعيل، عماد احمد،" خصائص نظم المعلومات المحاسبية و اثزها في تحديد خيار المنافسة الاستراتيجي في الادارتين العليا و الوسطى"، رسـالة

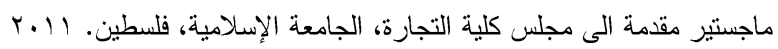
ـ ا. جعفر ، عبد الكريم، علي، سنية كاظم،" تاثير تقانة المعلومات في تحقيق الميزة التتافسية"، مجلة كلية بغداد للعلوم الاقتصادية الجامعة"، العـدد 9 ؛ـ. 


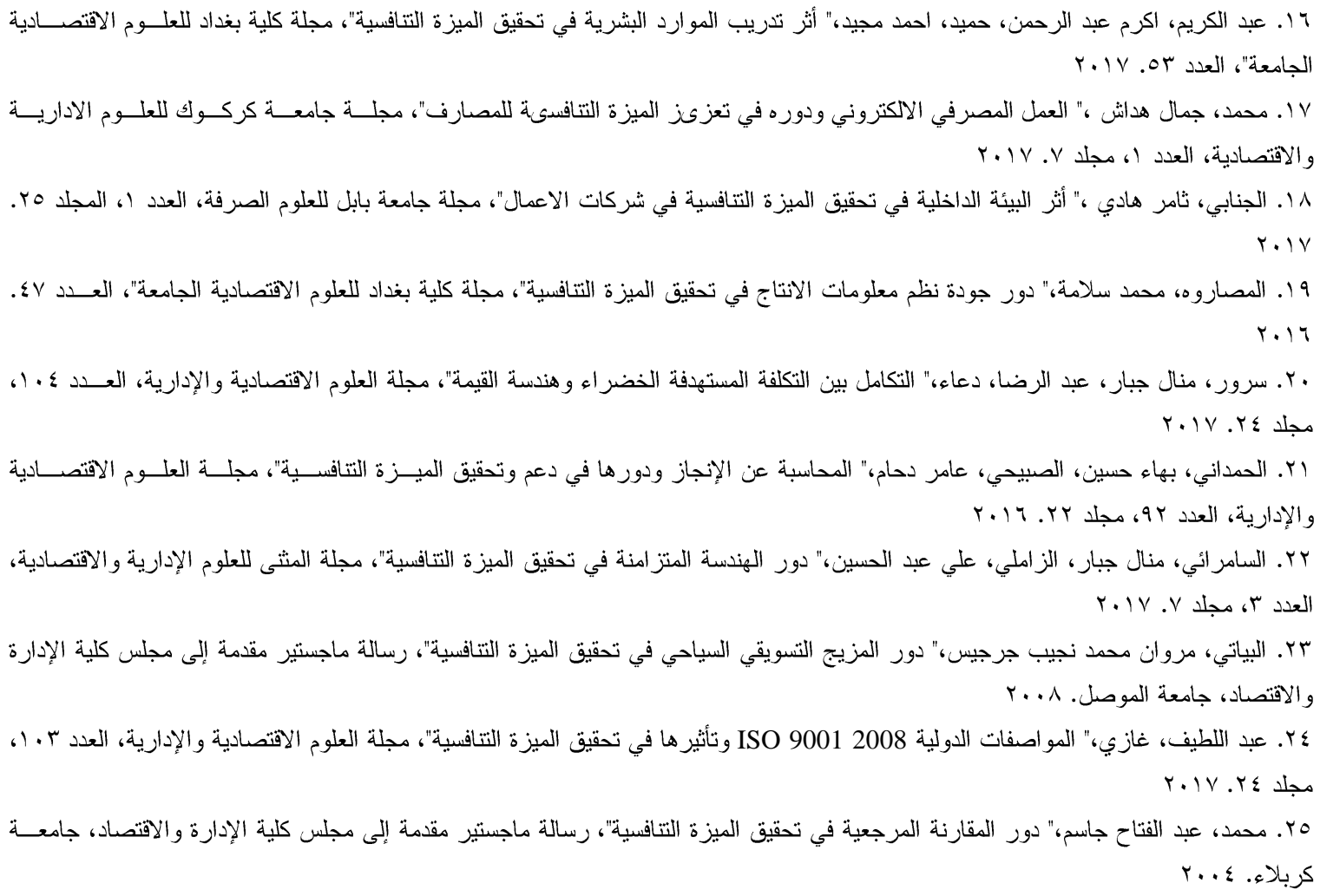

